\title{
Doing it the vendor's way: Running multiple subjects in reading experiments using Data General's Diskette Operating System
}

\author{
DAVID E. KIERAS \\ University of Arizona, Tucson, Arizona 85721
}

\begin{abstract}
Running multiple subjects on a laboratory computer is usually a difficult problem. An easy solution is possible in certain experimental domains by using Data General's software that allows multitask programming in FORTRAN. An example is provided, and performance of the system is discussed.
\end{abstract}

The problem often arises in using a laboratory computer in cognitive psychology experiments that, in order to attain maximum throughput and efficient use of laboratory time and assistants' wages, it is necessary to run more than one subject at a time. For such a system to be really practical, the subjects must be able to proceed through the experiment independently and at their own pace. In order to do this, some form of multitasking must be available on the computer. Such a multitasking facility can be obtained in three ways: (1) writing the application program so that it is "smart" enough to keep track of the multiple subjects, (2) writing a user-level large-scale programming system that provides a simple way to write multisubject programs, and (3) trying to make use of software systems provided by the computer vendor.

The smart program approach has the problem that such a program is likely to be very complicated; hence writing or modifying the program for a new experiment is difficult and tedious. The user-level system approach, represented by the GEPS system at the University of Michigan (Kieras, 1973), is a good solution except for the fact that a considerable investment in software development is required (cf. Kieras, 1976). Finally, the vendor-supplied software for multitask operation is usually not very suitable for multisubject experiment programming. This is because such software usually assumes multiple users, as in a timesharing computer facility. As a consequence, such systems often have inappropriate timing assumptions and cannot run adequately fast unless they are on a large fast machine with a hard-disk storage. Such machines are expensive. But in fact, multisubject running requires only a very simple form of multitask operation, far simpler than the multiuser sort of system. But few vendor-supplied systems provide multitasking at this simple level.

Thus setting up multiple-subject experiments on a laboratory computer is usually a difficult undertaking, especially if the computer is small and of moderate cost. This paper describes an attractive solution to the multiple-subject programming problem, which has been in active use in our laboratory at the University of Arizona for some time. Our machine is a low-cost Data General (DG) MicroNOVA, equipped with DG's Diskette Operating System (DOS). This vendor-supplied system has a multitasking facility that enables us to write multiple-subject running programs in FORTRAN quickly and easily. Although this system is hardly new, few psychologists are aware of its advantages.

\section{DESCRIPTION OF THE SYSTEM}

Our MicroNOVA is equipped with dual DG floppies, $28 \mathrm{~K}$ of MOS memory, and five asynchronous interfaces, one for the console terminal, one for driving the outboard hard-copy printer or linking to the campus PDP10 system, and three to drive subject station terminals. The subject terminals are Teleray Model $3811 \mathrm{~s}$ with upper- and lowercase, 24 by 80 character screens, and cursor addressing. They are run at 9,600 baud. The MicroNOVA CPU includes a real-time clock that runs on a $1.83105-\mathrm{msec}$ period, which is adequate for timing. A full set of analog hardware is being installed; since this is to be used in single-subject physiological experiments, it is not of concern in this paper. Although this system is powerful for doing experiments in verbal memory or prose comprehension paradigms, the computer hardware (not including the terminals) costs less than $\$ 12,000$.

The software system is the main concern of this paper. The DOS is a subset of DG's hard-disk real-time operating system, RDOS. It includes full file-handling utilities, a TECO-like file editor, an assembler, a macroassembler, an overlay-handling loader, and a FORTRAN IV compiler. The FORTRAN support for disk file $\mathrm{I} / \mathrm{O}$ is good; sequential, record-oriented, and direct-block access are provided. The system makes good use of disk space by allocating space for a file using blocks that can be scattered in noncontiguous locations on the disk. The key feature of the DOS system that makes multiple-subject programs easy to write is that the FORTRAN compiler generates 
Table 1

Main Task Program

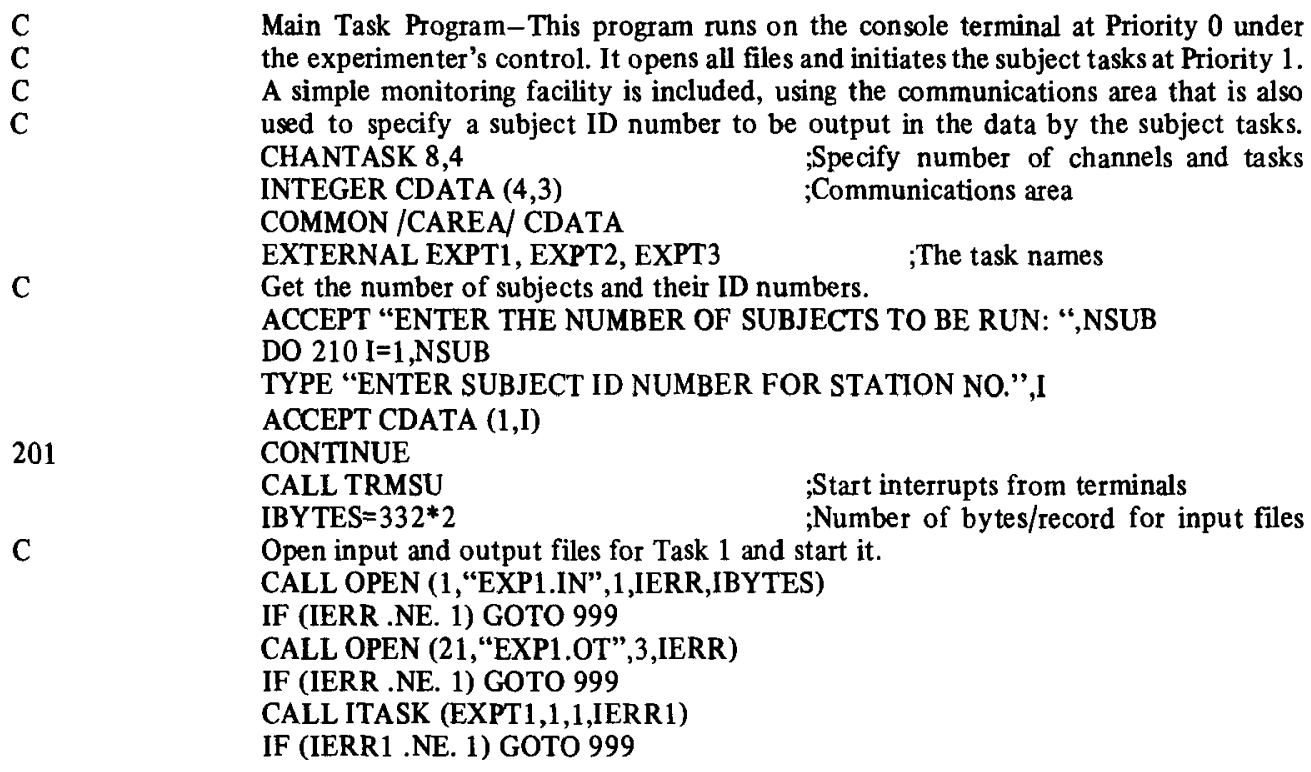

(Similar code here for opening files and starting Tasks 2 and 3)

9000

ACCEPT "ENTER 1 TO CHECK,9 TO STOP: “,ICOMMAND

IF (ICOMMAND .EQ. 1) TYPE CDATA

IF (ICOMMAND .EQ. 9) GOTO 9001

GOTO 9000

9001

CALL AKILL(1)

STOP

999 TYPE “ERROR IN SYSTEM CALL”

TYPE IERR,IERR 1

STOP

END

reentrant code. Thus, a single copy of a program can be shared by the tasks for the separate subjects, a central feature of such systems as GEPS.

\section{WRITING MULTIPLE-SUBJECT PROGRAMS}

Tables 1, 2, and 3 provide an example of a multiplesubject program for a reading experiment. To save space in this paper, the error-checking and monitoring code has been eliminated, and the procedure has been simplified from that actually used in experiments. This program collects reading times for individual sentences in short passages. Each passage consists of 11 short sentences. Each sentence is displayed on the screen of the subject's terminal. When the subject finishes reading the sentence, he or she taps the space bar and the sentence disappears, to be replaced by the next sentence. The program records in a disk file the time each sentence was left on the screen for reading. The sentence set for a passage is read in from a disk file in a 332-word record. The sentences themselves contain cursor control characters to erase the screen and position each sentence in the center of the screen. An off-line program, run before the session begins, is used to generate the proper sequence of sentences and passages.

A multiple-subject experiment program consists of a main task routine, three dummy routines (one for each subject), and an experiment subroutine, which is the

Table 2

Dummy Task Program

C Dummy program for Task 1. This program represents the
C task associated with the first subject station. It simply
C defines the task ID number and the input and output file
C numbers associated with this subject and calls the re-
C entrant subroutine common to all three tasks. The other
C tasks are represented by similar programs with appropriate
C task ID and file specifications.
TASK EXPT1
IDTASK=1
INFUN=1 ;Tummy program for Task 1
IOFUN=21 ; In number
Call the subject running subroutine with these parameters.
CALL EXPRS(IDTASK,INFUN,IOFUN)
CALL KILL
END


Table 3

Experiment Running Subroutine

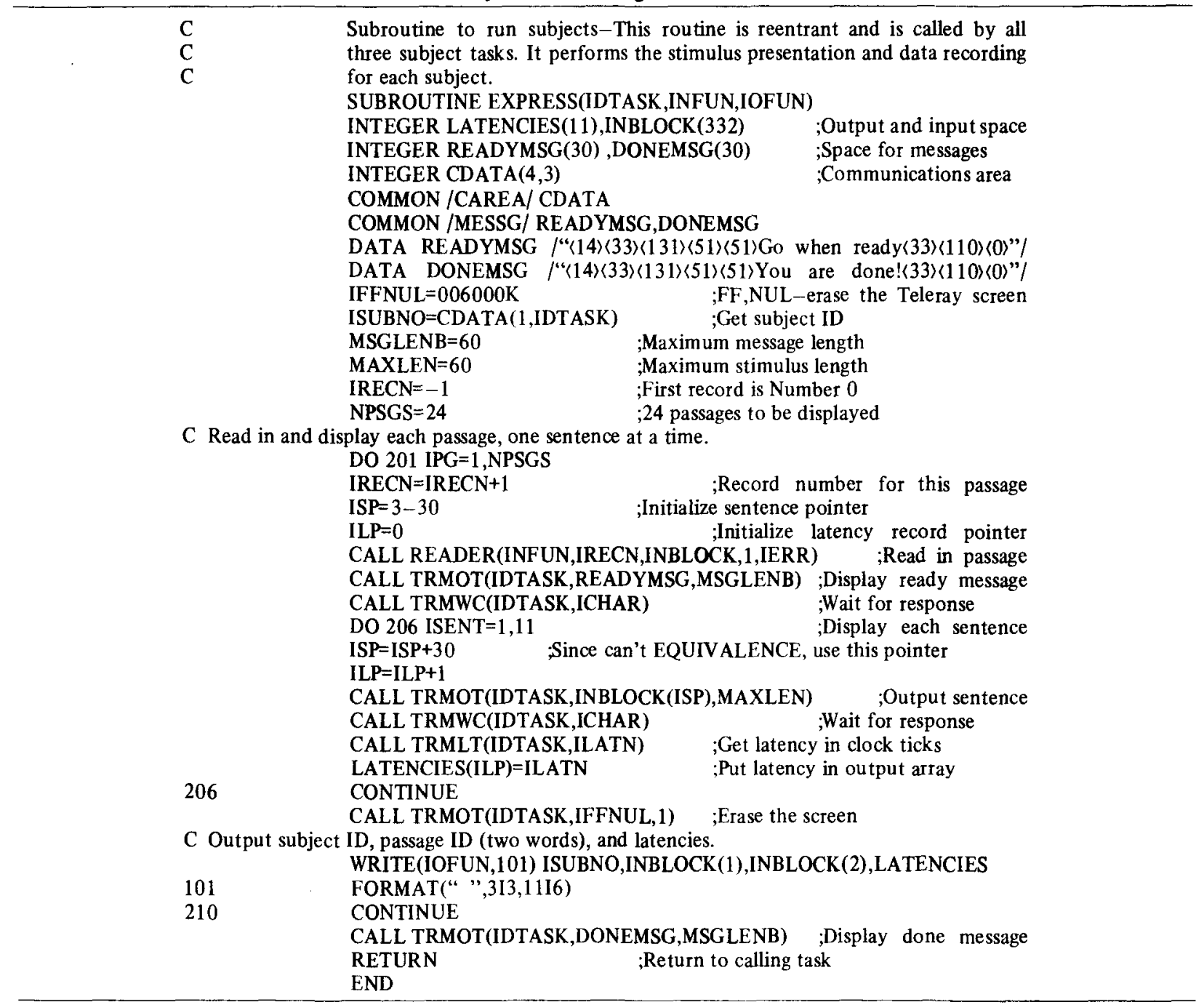

only routine specific to a single experimental procedure. The main task, shown in Table 1 , interacts with the experimenter, requesting the number of subjects and their identification numbers at the beginning of the session and providing on-line monitoring of the progress of each subject. When the experimenter is ready to begin, the main task opens the files for each subject's task and then initiates the three dummy subject tasks. The dummy tasks, illustrated in Table 2, do nothing more than call the experiment subroutine with parameters of the task number and the input and output file numbers. Since the FORTRAN run-time code for the experiment subroutine is reentrant, the same copy of it can be called from each of the subject tasks. This feature saves not only space but also a considerable amount of time and trouble, since modifications for the experiment are made by changing only the one subroutine.

Using the locally written subroutines TRMOT, TRMWC, and TRMLS, the experiment running subroutine, shown in Table 3 , does $1 / 0$ to the terminal corresponding to the task number. The messages defined in the DATA statements include cursor control codes to position the message. The subroutine takes stimulus materials from the specified input file, collecting responses and latencies and writing data to the designated output file. The DOS multitask executive suspends operation of tasks waiting on $\mathrm{I} / \mathrm{O}$, and allows the other tasks to proceed. Hence each subject is able to work independently of the others. When one subject finishes, his task is terminated, but the others are free to continue running.

\section{IMPLEMENTATION REQUIREMENTS}

In order to use the DOS system for running experiments, certain routines had to be written in assembler. The most important were the terminal handlers. The terminal handlers in the system were not useful, since they did not include the features required for experi- 
mentation such as time recording for latencies. Due to the simplicity of DG's hardware, these routines were rather easy to write.

The other subroutines were concerned with timing. A somewhat kludgy routine was written that picks up the system clock locations, which are updated by the MicroNOVA's built-in real-time clock. Another routine was written to provide a delay function in which a task could be suspended for a specified period of time. A system call for this function is present in the larger RDOS software, but for some inexplicable reason it has not been included in the DOS software.

\section{EVALUATION OF THE SYSTEM}

In any operating system of the complexity of DOS, there will be considerable system overhead, especially in trying to run a high-level language like FORTRAN in real-time and with reentrant code. In many applications, such as driving CRT displays at tachistoscopic speed, the system overhead would lead to unacceptable delays in processing. However, the performance of the system in our sentence memory and prose comprehension experiments is quite satisfactory. This particular experimental domain makes no stringent requirements on the time response of the machine. For example, the minimum response latencies that the system must work with, obtained in experiments in which simple passages are presented one sentence at a time, are fairly long, on the order of $1 \mathrm{sec}$. Other experiments use 1,600-character passages in which the display-filling time at 9,600 baud is itself between 1 and $2 \mathrm{sec}$, and reading times (time before the subject responds) are on the order of $1 \mathrm{~min}$.

In this setting, the CPU has plenty of time in which to perform multitask system functions. Since the terminal $\mathrm{I} / \mathrm{O}$, clock updating, and time recording are handled at priority interrupt levels, the system overhead does not slow down the presentation of a stimulus or interfere unduly with obtaining accurate reaction times. Furthermore, the standard video terminal possesses a substantial time indeterminancy introduced by the raster-scan process. For our laboratory applications, this problem is not a serious source of error, but it does have the effect of completely swamping the much smaller time errors introduced by the operating system overhead.

The major bottleneck in the system is the slowness of the floppy disks. However, using the multitasking facility allows the system to recover the disk access time, hence terminal $I / O$ and the experiment running subroutine can operate during disk access time. Thus, the disk does not introduce serious timing problems, but the experiment subroutine logic has to be planned to perform disk $\mathrm{I} / \mathrm{O}$ at noncritical times for the subject, such as between blocks. In this way, response of the system to the subject is very fast within the block, but can be delayed for 1 to $2 \mathrm{sec}$ during the noncritical time between blocks while the program is bringing in the next batch of stimulus material. In these experiments, each block consists of a passage; some delay between passages is included in the procedure anyway, so there is no problem with this approach.

Thus far, the only design flaw in the system that affects the ease of the FORTRAN programming concerns a restriction on the use of EQUIVALENCE statements in the experiment subroutine. Due to the stack allocation algorithm, EQUIVALENCE statements must refer to variables in COMMON. However, all variables in COMMON are implicitly known to all tasks. It is highly desirable to write the experiment subroutine in such a way that as little reference as possible must be made to the task number. Hence, having the subroutine keep track of separate areas in COMMON for each task would be undesirable. The best way to define variables in the experiment subroutine is as stack variables, which occupy space on the stack for each task. EQUIVALENCE statements effectively cannot be used in the experiment subroutine.

\section{CONCLUSION}

Being able to write experiment programs in FORTRAN is a great advantage, due to the familiarity of this language. Students can apply their knowledge from introductory programming courses to the programming of experiments on the laboratory computer. The primary obstacle facing the busy researcher and the novice student, the complexity introduced by running multiple subjects, largely disappears when the DOS multitasking facility is used. The overall result is a system that is almost as easy to use as a large-scale user-level system of the GEPS type, but that requires very little effort to bring up, since DG's system provides all of the hard-to-write components. Doing it the vendor's way actually results in highly efficient and effective laboratory computer software.

\section{REFERENCES}

KiERAS, D. A. A general experiment programming system for the IBM 1800. Behavior Research Methods \& Instrumentation, 1973, 5, 235-239.

KIERAs, D. Programmer vs. system: The issues. Behavior Research Methods \& Instrumentation, 1976, 8, 174-177. 